Denmark, and Iceland. The cases were collected during one and a half years, beginning 1 July 1997.

Method-Clinical variables (including those constituting the EULAR and ILAR criteria) and laboratory findings were registered longitudinally for each patient by paediatricians experienced in paediatric rheumatology. A database was constructed specifically for this purpose. In this study clinical findings and laboratory data from the first 6 months (for certain variables also for the first 12 months) were considered.

Results - 322 patients qualified for classification according to the ILAR criteria. Of those 322 patients, 291 had a disease duration of $>3$ months and were classified according to the EULAR criteria. Thus, 31/322 (10\%) children were diagnosed only according to the ILAR criteria. $15 \%$ of the patients did not fulfil ILAR criteria for any of the categories and $6 \%$ fulfilled criteria for more than one category. As an example of the effect of different classification systems in our study, 160/ 322 were classified as oligoarticular by ILAR criteria and $184 / 291$ by EULAR criteria.

Conclusion-The qualifications for the ILAR criteria are different from those of the EULAR criteria, which makes simple comparison neither possible nor scientifically relevant. One possibility is to study the construct validity of the respective criteria to give us a better idea of the classification and its consequences.

\subsection{Juvenile spondyloarthropathies: checking criteria for diagnosis and classification at the start and after 6} months of disease

J DEHOORNE, B VANNEUVILLE R JOOS, J VANDE WALLE, EMVEYS Centre for Paediatric Rheumatology, University Hospital, Gent, Belgium

Classification of juvenile spondyloarthropathies remains a "critical" point in paediatric rheumatology.

Objective - To validate classification methods in a university hospital treated population by a retrospective cross sectional study.

Methods - Four sets of criteria especially designed for juvenile patients (GarmischPartenkirchen-JSA, SEA syndrome, enthesitis related arthritis, atypical spondarthritis) and two sets of criteria for patients without age specification (ESSG, Amor) were checked in a group of 20 patients at the start of disease and after six months.

Statistics-The sensitivity and specificity were calculated as well as the reproducibility after six months.

Results - The preliminary results show equal sensitivities for all sets except for the SEA syndrome criteria (which was the lowest). Although in some patients a switch to other types of juvenile chronic arthritis occurred, the initial fulfilment of the criteria was highly predictive for the fulfilment after six months.

\section{Connective tissue disorders}

3.1 Analysis of Raynaud's phenomenon in an infant and teenage population

ML GÀMIR, MJ RUBIRA,

CM VAZQUEZ, M REVENGA, R HORTAL

Rheumatology Service, Hospital Ramòn y

Cajal, Madrid, Spain

Objectives-To analyse the onset and evolution patterns as well as the prognostic value of Raynaud's phenomenon (RP) in a cohort of children diagnosed and followed up in our paediatric rheumatology service.

Material and methods - In a prospective study several clinical, analytical, and outcome variables were collected from a group of patients under the age of 18 years who were admitted to our department because of a history of positive RP.

Results -54 patients ( 47 female, 7 male) with a mean age of onset of the disease of 13.5 years (range 2-18) were studied. In 31 patients $(57 \%) \mathrm{RP}$ occurred in isolation and in 23 patients $(43 \%)$ it coexisted with arthritis, subcutaneous nodules and/or hand oedema.

After an average evolution period of 7 years (range 1-14) 10 patients $(19 \%)$ developed a defined connective tissue disease (CTD) (4 mixed connective tissue diseases, 3 scleroderma, 2 systemic lupus erythematosus, 1 juvenile chronic arthritis), 31 patients $(57 \%)$ presented some feature of undifferentiated CTD (arthritis, hand oedema, nodules, finger ischaemia, livedo, mucose ulcers, iridocyclitis, positive antinuclear antibodies (ANA), and pathological capillaroscopy pattern). 13 patients $(24 \%)$ remain currently as primary RP.

Conclusions-(1) Over 50\% (75\% in our case) of patients with paediatric onset RP, eventually develop a CTD or present features consistent with this kind of disorder.

(2) The greater proportion found in our study might be explained by the selection of patients examined.

(3) Arthritis, hand oedema, co-occurrence of ANA and the presence of findings in nail capillaroscopy, have a good prognostic value.

3.2 Severe cardiac disease among children with diffuse cutaneous systemic sclerosis (SSc) and polymyositis: report of 4 cases

P QUARTIER, D BONNET, JC FOURNET, C BODEMER, PH ACAR, M OUACHÉE, AM PRIEUR

Immuno-hématologie, Cardiologie and

Dermatologie pédiatrique, Hôpital

Necker-Enfants malades, Paris, France

Patients and methods-We retrospectively reviewed the charts of 4 girls who met the ACR criteria for diffuse cutaneous SSc and had polymyositis, as defined by the presence of proximal muscle weakness and raised serum creatine phosphokinase level. Disease duration and age on admission ranged from 4 to 12 months and from 7 to 13 years respectively. Dysphagia and oesophageal dysmotility were present in 3 patients, arthritis in 2 , lung restrictive syndrome in 2 , and echocardiographic anomalies in 2 , including marked non-obstructive cardiomyopathy (NOCM) in 1 child. One patient secondarily developed severe NOCM and bowel disease.

Results-Treatment with cyclosporin, methotrexate, and corticosteroids (including pulsed intravenous methylprednisolone in 3 cases) improved the skin thickness score, muscle weakness, arthritis, and lung restrictive syndrome. Oesophageal dysmotility and bowel disease did not improve. The 2 patients who had severe NOCM before the triple combination treatment died after 19 and 31 months. Severe NOCM developed in a third patient 6 months after treatment onset. Cardiac transplantation was performed and the child is alive and well 3 months after. One child treated with cyclosporin only is alive after a follow up of 48 months.

Conclusions-As previously described in adults, children with SSc and myositis may be particularly prone to develop severe NOCM.
Earlier and more intensive treatments are probably required. In the case of yet constituted severe NOCM, heart transplantation should be considered.

3.3 Diagnosis of Sjögren's syndrome in childhood

AG CLEARY^, JA SILLS ${ }^{\star}$, E BAILDAM†, JE DAVIDSON ${ }^{\star}$

${ }^{\star}$ Royal Liverpool Children's Hospital, UK; †Royal Manchester Children's Hospital, UK

Sjögren's syndrome is rare in childhood. We have recently seen 5 children with definite and two with possible Sjögren's syndrome whose investigations highlighted some of the difficulties in diagnosis in the paediatric age group.

Of the children with definite Sjögren's syndrome one had mixed connective tissue disease (MCTD), one juvenile dermatomyositis, and one systemic lupus erythematosus. Of those with possible Sjögren's one has MCTD. The others have no definite underlying diagnosis.

We used the European classification of Sjögren's syndrome as modified by Vitali et al $1993^{1}$ to assess these patients and rapidly became aware of difficulties in using these criteria in children.

Four had symptoms of dry eyes, and six had dry eyes on ophthalmological testing. Three have had symptomatic dry mouths and two have had low measured basal salivary flow rates. There are, however, no validated age reference standards for tear and salivary flow rates in childhood.

Sialography, scintigraphy, and histology are used in the classification. Lip biopsy is unacceptable to most children. One patient had a lip biopsy, which proved unhelpful. We have found ultrasound to be well tolerated and to give information comparable with that obtained by magnetic resonance scanning or sialography. Parotid scintigraphy was performed in two children and thought to be abnormal, but interpretation was hampered by the lack of reference standards in childhood.

Investigation of children with possible Sjögren's syndrome in our units has highlighted problems with applying the current diagnostic criteria in childhood. There is a need for age appropriate criteria to be validated for use in childhood.

1 Vitali C, Bombardieri S, Moutsopoulos HM, Balestrieri G, Bencivelli W, Bernstein RM, et al. Preliminary criteria for the classification of Sjögren's syndrome. Results of a prospective concerted action supported by the European Community. Arthritis Rheum 1993;36:340-7.

3.4 Life experiences of adolescents living with systemic lupus erythematosus

CHM SILVA, W MACHADO, MHB KISS

Department of Paediatrics, School of Medicine,

Federal University of Uberlândia and

University of São Paulo, Brazil

Systemic lupus erythematosus (SLE) is a severe chronic disease commonly associated with severe personal, social, and familiar repercussions mainly in adolescents. To get to know the way in which these patients perceive and compare themselves according to the changes and the treatment the disease imposes on their routines, monthly meetings with adolescents with a diagnosis of SLE without central nervous system disease have taken place. Identification of the interests of the group of adolescents was possible through discussion and psychodrama techniques conducted by a doctor and by a social assistant with a degree in psychodrama. The 
first concerns referred to by the adolescents were the side effects of steroid treatment (obesity and short stature), the difficulty of the daily use of solar protectors, the limitation on physical activity, the dietary restrictions, how to talk about the disease to friends and relatives, and the constant need for examinations and testing and, eventually, for invasive procedures (biopsies). The adolescents requested information about the disease itself, its treatment, and the possibility of cure, pregnancy, and contraceptive use. The authors emphasise the importance of the methodology with groups of adolescents with SLE, in which the partner works as a "mirror"-making the adolescents perceive themselves, and as a facilitator-helping them to identify their concerns, anxiety, and expectations. The group was shown to be effective in complementing medical assistance, promoting better adherence to the treatment and a better social and familiar environment through the explanation of several aspects of the disease.

\subsection{Neutropenia caused by anti-Ro} autoantibodies in neonatal lupus

\section{R CIMAZ ${ }^{\star}, \mathrm{H}$ SCOFIELD +}

*Paediatrics, ICP, Milano, Italy; fOklahoma Medical Research Foundation, OK, USA

Background-Neonatal lupus (NL) provides a situation in which the pathogenicity of antibodies can be evaluated. Transplacentally acquired anti-Ro (or SS-A) autoantibodies are found in the sera of babies with congenital heart block or other less common manifestations of NL, such as skin rash, liver disease, or haemocytopenia.

Case report-We report an infant, born to a mother with anti-Ro, who had profound neutropenia. A 30 year old woman with an undifferentiated connective tissue disease gave birth to a healthy girl. Laboratory tests performed on the baby at follow up, 10 weeks after birth, showed normal white blood count, but an absolute neutrophil count of $120 / \mu 1$. Causes of congenital and acquired neutropenia were excluded; a bone marrow aspirate did not show major abnormalities. IgG antigranulocyte antibodies were detected in the baby's serum. Fluorescence activated cell sorting showed binding of mother's and baby's serum to intact neutrophils. To determine the specificity of this binding, purified $60 \mathrm{kDa}$ Ro was used as inhibitor. After this treatment, the binding of each serum to the cell surface of neutrophils was drastically reduced. The specificity of binding was further studied in immunoblot and enzyme linked immunosorbent assay (ELISA). When HeLa cell extract was used as antigen, sera of both the mother and child bound $48 \mathrm{kDa}$ La but $52 \mathrm{kDa}$ Ro was not bound. The $60 \mathrm{kDa}$ Ro antigen was bound strongly by both sera in a direct antigen solid phase assay. Using nitrogen cavitation, we purified neutrophil membranes and found that the mother's and child's sera bound a species at a predicted molecular weight of $64 \mathrm{kDa}$, recently recognised as a novel autoantigen in systemic lupus erythematosus and immunologically cross reactive with $60 \mathrm{kDa}$ Ro. Such binding was in fact inhibited by incubation of sera with $60 \mathrm{kDa}$ Ro.

Conclusion-Our results show that anti-Ro can directly lower the neutrophil count by binding the cell surface of neutrophils.
3.6 Laboratory markers of endothelial damage in the assesment of the activity of childhood rheumatic diseases

P DOLEZALOVA, P TELEKESOVA,

D NEMCOVA, J HOZA

Department of Paediatrics and Adolescent Medicine, Charles University in Prague, 1 st

Faculty of Medicine, Czech Republic

Various laboratory markers of endothelial cell damage/activation seem to play an important part in rheumatic disease pathogenesis. Among these we have chosen factor VIII related antigen (vWF) and soluble forms of adhesion molecules ICAM-1 and E-selectin (E-sel) and measured their blood levels in 110 children aged 1-18 years (mean 11.1) with the following diagnoses: polyarticular juvenile idiopathic arthritis (JIA) (JIA-poly, $\mathrm{n}=18$ ), oligoarticular course JIA (JIA-oligo, $n=14$ ), juvenile dermatomyositis (JDM), systemic sclerosis $(\mathrm{SSc})$, and overlap syndromes $(\mathrm{SSc} /$ JDM, $n=11$ ), systemic lupus erythematosus $(S L E, n=6)$, primary vasculitides (VAS, $n=9$ ), Henoch-Schönlein purpura (HSP, $n=11$ ), acutely ill febrile children $(\mathrm{n}=10)$, and healthy controls $(n=31)$. Non-specific inflammatory variables (erythrocyte sedimentation rate, $\mathrm{C}$ reactive protein) and joint counts (where appropriate) were recorded at the same time.

vWF reached the highest levels in patients with SLE (mean (SD) 159.9 (24.3)\%, $\mathrm{p}=0.05$ ), whereas, interestingly, it was within the reference limits in $77.8 \%$ of patients with JDM/SSc and $82.4 \%$ of patients with VAS. We found a significant tendency for both ICAM-1 and E-sel to decrease with age $(p=0.01)$. ICAM-1 reached the highest values in the VAS $(428.4(113.4) \mathrm{ng} / \mathrm{ml})$ and JIA-poly (376.51 $(22.6) \mathrm{ng} / \mathrm{ml})$ groups $(\mathrm{p}=0.05)$. Patients with acute HSP did not differ significantly from controls. In the JIA-poly group E-sel levels also were significantly higher than in controls (144 (82.7) $\mathrm{ng} / \mathrm{ml}, \mathrm{p}=0.05$ ). All patients in this group had clinically active disease; its duration and current treatment did not have any significant impact. No signs of secondary vasculitis were seen and there were only 2 patients with systemic onset JIA. High adhesion molecule values could be a consequence of generalised endothelial activation followed by their nonspecific release. Possibly, also, hypertrophic and highly vascular synovium in inflamed joints could be the main source of their production. To answer this question we examined 14 paired samples of sera and synovial fluid from patients in both JIA groups where joint aspiration was indicated. Interestingly, we found no significant difference in ICAM-1 and E-sel concentrations.

vWF and circulating adhesion molecule levels bring interesting insights into the pathogenesis of endothelial activation and damage processes, but their clinical usefulness and disease specificity need to be studied further.

Supported by the Charles University Grant Agency, grant No 202/98.

3.7 Case report of a patient with catch 22 sequence: haematological and bony abnormalities; the chronic arthritis responds to etanercept

HI BRUNNER ${ }^{\star}$, J TAYLOR*, A GROM*, E SCHORRY†, MH PASSO ${ }^{\star}$

Children's Hospital Medical Center, ${ }^{\star}$ Division of Rheumatology and TGenetics, Cincinnati, USA Background-Children with some genetic abnormalities are known to have an increased incidence of chronic arthropathy. This is also true for patients with deletions of chromosome 22q11.2. We present a patient with a large deletion on chromosome 22q11.2 and yet unreported combination of symptoms. Case report-This girl presented with seizures in the neonatal period in association with hypocalcaemia due to hypoparathyoidism. She had a submucosal cleft palate and was later found to have structural temporomandibular joint abnormalities, bilaterally subluxed radial heads, posterior fusion of the cervical vertebrae at level C2-3, and abnormally shaped foramen magnum and clivus. The cardiac evaluation showed a right aortic arch and an abnormal left superior vena cava draining into the coronary sinus. At the age of 3 years the patient developed Coombs positive haemolytic anaemia and thrombocytopenia. Her haematological symptoms were well controlled with low dose steroids every other day. During flares, which occurred every 3-6 months, high dose steroids were used for treatment. At the age of 13 years the patient was diagnosed with antinuclear antibody negative, rheumatoid factor negative, erosive polyarticular arthritis, which was poorly responsive to steroids and methotrexate. Remission of arthritis was induced with etanercept. However, the start of etanercept coincided with worsening of the previously observed haematological symptoms and with bone marrow suppression.

Conclusions-Compared with the normal population the risk of chronic arthritis is 150 times higher for patients with deletion at 22q11.2, and about $10 \%$ of these patients have haematological abnormalities. Thus the gene region 22q11.2 may code for susceptibility genes of autoimmune diseases and chronic arthritis. Etanercept may be beneficial for the treatment of other arthropathies seen with genetic syndromes.

\subsection{Assessment of damage in juvenile} onset systemic lupus erythematosus

A RAVELLI, C DUARTE-SALAZAR, S BURATTI, N RUPERTO, A PISTORIO, D MIGLIAVACCA, V GERLONI, F FALCINI, F CORONA, L LEPORE, G ALPIGIANI, MR MALDONADOVELAZQUEZ, R BERINSTAINMANTEROLA, R BURGOS-VARGAS, RS RODRIGUEZ SUAREZ, A REIFF, K GALLAGHER, B SHAHAM, B BERNSTEIN, J LEMIRE, S ALBANI, A MARTINI

IRCCS Policlinico S Matteo-University of Pavia and Italian Paediatric Rheumatology Study Group, Italy; Hospital General and Infantil de Mexico, Mexico; Childrens Hospital Los Angeles and UCSD Medical Center San Diego, USA Objective - To investigate the prevalence of accumulated damage in patients with juvenile onset SLE and the relation between damage and demographic and clinical variables, overall disease activity, quality of life, and cumulative drug treatments.

Methods-A prospective, cross sectional, multinational study was used. Inclusion criteria: (a) diagnosis of SLE by ACR criteria; (b) onset age $<18$ years; (c) disease duration $\geqslant 12$ months. Instruments used: SLICC/ACR Damage Index (SDI), SLEDAI, Child Health Questionnaire.

Results -218 patients were enrolled -85 from Italy, 73 from USA, and 60 from Mexico. $86 \%$ were female, with a mean onset age of 11.4 years and a mean disease duration of 5.8 years. $150(69 \%)$ patients had damage. The mean (SD) SDI score was $1.6(1.8)$, range $0-12$. The organ systems more commonly affected by damage were renal $(23 \%)$, skin (21.7\%), neuropsychiatric (NPS) (17.5), and ocular (9.7\%). Logistic regression analyses 
showed that the presence of damage was positively associated with the SLEDAI score (odds ratio $(\mathrm{OR})=1.1, \mathrm{p}=0.005)$, cumulative drug score $(\mathrm{OR}=1.0, \mathrm{p}<0.001)$, and NPS disease at onset $(\mathrm{OR}=4.4, \mathrm{p}=0.008)$ and negatively associated with Raynaud phenomenon at onset $(\mathrm{OR}=0.2, \mathrm{p}=0.01)$.

Conclusions-Our results show that permanent damage is common in paediatric onset SLE and is significantly associated with lupus disease activity and cumulative drug treatment. NPS disease at onset is a strong predictor of late damage, whereas the presence of Raynaud phenomenon may exert a protective effect.

\subsection{Uncommon causes of liver disease in juvenile systemic lupus erythematosus}

A RAVELLI, C MALATTIA, F TEMPORINI, S MAGNI MANZONI, C MORETTI, G ROSSI, L BASSI, A MARTINI

Dipartimento di Scienze Pediatriche, Università di Pavia, IRCCS Policlinico San Matteo,

Pavia, Italy

Background-Severe liver disorders are rare in patients with systemic lupus erythematosus (SLE). We report two patients with juvenile SLE who developed uncommon hepatic complications.

Case reports-Patient No 1: A 10 year old girl was admitted with a 3 week history of malar rash, intermittent arthritis, fatigue, and abdominal pain. Investigations showed haemolytic anaemia, thrombocytopenia, and positive antinuclear antibodies (ANA) and anti-DNA antibodies. High titre anticardiolipin antibodies (aCL) and lupus anticoagulant (LA) were detected. A diagnosis of SLE was established. During a stay in hospital the girl developed enlargement of the liver, which was tender at palpation. Ultrasonography showed a large thrombus occluding the lumen of the inferior vena cava (Budd-Chiari syndrome). Based on the association of vascular thrombosis and positive antiphospholipid antibodies, antiphospholipid syndrome was diagnosed and long term anticoagulation treatment was started.

Patient No 2: A 9 year old girl, who had had autoimmune thyroiditis since the age of 6 years, was admitted with a 1 week history of fever, fatigue, arthromyalgia, and purpuric rash in the legs. Physical examinations showed hepatosplenomegaly, ascites, and oedema of the ankles. Laboratory investigations disclosed pancytopenia, liver transaminase increase, hypoalbuminaemia, low serum complement levels, and positive ANA and anti-DNA antibodies. aCL and LA were absent. Liver ultrasonography showed an enlarged liver with diffuse pseudonodular changes. A percutaneous biopsy showed necrotising arteritis with nodular regenerative hyperplasia of the liver. A diagnosis of SLE with necrotising arteritis of the liver was made, and treatment with high dose prednisone and cyclophosphamide was begun.

Conclusion-The reported patients demonstrate the protean clinical spectrum of liver disease in juvenile SLE.

\section{Epidemiology and outcome}

4.1 Epidemiology of rheumatological diseases in Latvia

V STANEVICHA, A KOKINA, R SHANTERE, A SHEGOLEVS Medical Academy of Latvia

Objective-To research the epidemiology of rheumatic diseases in children and adolescents aged 1-18 years to obtain comparative data with those of other countries.
Methods-We used the children's rheumatic diseases register data obtained by 1 January 2000 to determine incidence and prevalence. Results-1251 children and adolescents aged 1-18 have rheumatic diseases. $70.4 \%$ have been diagnosed juvenile chronic arthritis (JCA), with prevalence 165.1/100 000 and incidence $34.3 / 100000.36 \%$ have registered oligoarthritis, $61 \%$ polyarthritis (rheumatoid factor positive $5.2 \%$ ), $2 \%$ systemic form arthritis, $0.5 \%$ psoriasis with arthritis, $0.5 \%$ Crohn's disease and ulcerous colitis with arthritis. Eye injuries in patients with JCA occur in $2.4 \%$ of cases. The prevalence of collagenoses is $11.1 / 100000$ and incidence $2.1 / 100000$. Recent years show an increase of scleroderma with a prevalence 4.5/ 100 000. Since 1993 cases of rheumatic fever have increased, reaching a prevalence of $12.6 / 100000$ and an incidence of 2.1 100000 in $1999.49 .3 \%$ have endocarditis. JCA and collagenoses affect mostly female children, rheumatic fever occurs mostly in male children. Patients with acute arthritis have an incidence of 10.3/100 000. EpsteinBarr virus (9.8\%) and streptococcal infection $(8.6 \%)$ are most common.

Conclusion-Epidemiological data in children and adolescents with JCA and collagenoses are similar to those of the developed countries, except for a smaller occurrence of eye injuries in patients with JCA. Rheumatic fever with frequent valvular injuries has a comparatively higher occurrence. In $38 \%$ of patients the cause of acute arthritis is unknown.

\subsection{High prevalence of childhood chronic arthritis among the Shipibo people of Amazonian Peru}

M ANDERSSON, S MYRNERTS, A HANSSON, A FASTH

Department of Paediatrics, University of

Göteborg, Göteborg, Sweden

Few prevalence figures of childhood chronic arthritis exist for developing countries and even fewer for the indigenous population living a traditional life in those countries. We undertook a prospective population based study of the prevalence rate of childhood arthritis among the Shipibo people living in the Amazonian part of Peru.

Methods-As most adults and children are illiterate and time is vaguely defined as the number of elapsed rainy or dry seasons, no established criteria could be used. Instead a questionnaire was constructed with questions that aimed at differentiating between joint disorders of traumatic, infectious, and rheumatic aetiology. The questionnaire was given orally by an interpreter to all children present in the villages at time of the investigation and to a close adult relative. All children were physically examined for the presence of arthritis. The study area was Shipibo villages accessible from a smaller town, and the study was performed during January and February 1999

Results - 651 of 785 children (83\%) living in 6 areas of Yarina Cocha and 2 villages were examined personally by two of us and had the questionnaire applied. $4.3 \%$ had joint complaints of traumatic cause, $1.4 \%$ of possible infectious origin, and $1.8 \%$ a possible rheumatic joint disorder. Of the 12 children with a possible chronic arthritis, 4 had active arthritis at time of investigation, which corresponds with a prevalence of 614 per 100000 children $<16$ years of age. If all 12 children with a possible arthritis are included the prevalence is 1843 per 100000 children $<16$ years of age. This very high prevalence must be viewed with caution as the study period was short, the most remote part of the Shipibo area could not be accessed, the concept of medicine is traditional, and longstanding reactive arthritis might have been included. This means that the prevalence as well as possibly being overestimated might even have been underestimated. Also among adults of the Shipibo people a very high prevalence, close to $10 \%$ has been reported, indicating that the prevalence of chronic arthritis among children also may be high.

\subsection{Adaption and application of child} health assessment questionnaire (CHAQ) to an indigenous population of Amazonian Peru

S MYRNERTS, M ANDERSSON, A HANSSON, A FASTH

Department of Paediatrics, University of

Göteborg, Göteborg, Sweden

The CHAQ has been successfully adapted and applied to most industrialised societies and also to a country in transition like Costa Rica. Common to all those countries is a high educational level. We attempted to determine whether it was possible to adjust the Costa Rican version of the CHAQ (CR-CHAQ) to the conditions and language of the Shipibo people, a well defined indigenous group living a traditional life in the Amazonian part of Peru. Method-A cultural adaptation and linguistic translation of certain key words of the CR-CHAQ was done. It was tested on children and parents without joint disorders and was with minor alterations well understood. Self administration was not possible, however, as most adults and children cannot read or write. Instead an interpreter gave the Shipibo-CHAQ (SH-CHAQ). The SHCHAQ was tested on 12 children with possible rheumatic joint disorders, and a close adult. For children below 10 years of age, the SH-CHAQ was applied only to the adult. Results - Cross cultural validity: Most questions had to be adapted to Shipibo lifestyle and traditions. The children gave higher disability scores than the adults. Test-retest: A high correlation was found between the disability index for the first and second application both for children and adults. The correlation for children (Spearman $\left.r_{\mathrm{s}}=0.994, \mathrm{p}<0.002\right)$ was higher than that for the adults $\left(r_{\mathrm{s}}=0.839, \mathrm{p}<0.02\right)$. Interobserver score correlation: No correlation was found between the disability index for children and adults at the first application, but the second application showed a good correlation $\left(r_{\mathrm{s}}=0.975, \mathrm{p}<0.02\right)$. The difference between the first and second application might be due to exaggeration by parents who expected to obtain health care if the child was more severely ill. Conclusion-By carefully analysing the CHAQ and adapting the questions to the Shipibo culture we could successfully apply the new SH-CHAQ with a high test-retest and interobserver reliability.

4.4 Remission in juvenile chronic arthritis: a cohort study of 683 consecutive cases

\section{ARNOLDI, V GERLONI,} M GATTINARA, E LUPI, F FANTIN

Chair of Rheumatology of the University of

Milan, Centre for Rheumatic Children, Gaetano

Pini Institute, Milan, Italy

Evaluation of the prognosis of the juvenile forms of chronic arthritides is difficult because most patients are lost at follow up when they reach adulthood. Our institute is a privileged observatory as it is open to both juvenile and adult patients, so that children do 